\title{
Inviscid Limits and Regularity Estimates for the Solutions of the 2-D Dissipative Quasi-geostrophic Equations
}

\author{
JIAHONG WU
}

\begin{abstract}
We discuss two important topics of turbulence theory: inviscid limit and decay of the Fourier spectrum for the 2-D dissipative quasi-geostrophic (QGS) equations. In the first part we consider inviscid limits for both smooth and weak solutions of the 2-D dissipative QGS equations and prove that the classical solutions with smooth initial data tend to the solutions of the corresponding non-dissipative equations as the dissipative coefficient tends to zero. Here the convergence is in the strong $L^{2}$ sense and we give the optimal convergence rate. For the weak solutions of the dissipative QGS equations with $L^{2}$ initial data, we obtain weak $L^{2}$ inviscid limit results. In the second part we use the methods of Foias-Temam [8] and Doering-Titi [7] developed for the Navier-Stokes equations to establish exponential decay of the spatial Fourier spectrum for the solutions of the dissipative QGS equations, but we treat general norms, and also our method of estimating the nonlinear terms is different.
\end{abstract}

1. Introduction. We consider the 2-D surface quasi-geostrophic (QGS) equations

$$
\frac{\partial \vartheta}{\partial t}+u \cdot \nabla \vartheta+\kappa(-\Delta)^{\alpha} \vartheta=0, \quad 0 \leq \alpha \leq 1,
$$

where $\kappa>0$ in the case of the dissipative equations and $\kappa=0$ for the nondissipative equations. Here the velocity $u=\left(u_{1}, u_{2}\right)$ is determined from $\vartheta$ by a stream function $\psi$ :

$$
\left(u_{1}, u_{2}\right)=\left(-\frac{\partial \psi}{\partial x_{2}}, \frac{\partial \psi}{\partial x_{1}}\right)
$$

where $\psi$ satisfies

$$
(-\Delta)^{1 / 2} \psi=-\vartheta
$$


Equation (1) is a special case of the general quasi-geostrophic equations [10] for nonhomogeneous fluid flow in a rapidly rotating 3-D half-space with small Rossby and Ekman numbers, and its derivation involves assumptions of constant potential vorticity in the interior and constant buoyancy frequency [12]. Actually, the derivation is for $\alpha=1 / 2$, and the general fractional power $\alpha$ is considered in order to observe the minimal power of the Laplacian necessary in the analysis, and thus make a comparison with the 3-D Navier-Stokes equations. The variable $\vartheta$ is the potential temperature and $u$ is the fluid velocity. The dissipation coefficient $\kappa$ depends on viscosity and on the Rossby number, and tends to zero as viscosity tends to zero.

These equations have been under intensive investigations because of mathematical importance and potential applications in meteorology and oceanography ([3], [9], [10]). As pointed out in [3], the 2-D dissipative and non-dissipative QGS equations are strikingly analogous to the 3-D Navier-Stokes and the Euler equations. Inviscid limit results for the Navier-Stokes equations with smooth and rough initial data have been established ([1], [2], [4], [5], [6]). Naturally, the inviscid limit problem for the solutions of the dissipative QGS equations arises and, so far, we have seen no work in this direction.

We know from [3] that the QGS equations (defined on $\mathbb{R}^{2}$ or $\mathbb{T}^{2}$ ) with smooth initial data admit unique classical solutions for short times, and that the quantity

$$
\int_{0}^{T}\|\nabla \vartheta(\cdot, s)\|_{L^{\infty}} d s
$$

is responsible for possible singularity formation. The control of this quantity is also important in our proof of the inviscid limit results for smooth solutions.

Both dissipative and non-dissipative QGS equations on $\mathbb{T}^{2}$ with $L^{2}$ initial data have weak solutions in the distribution sense ([11]). Inviscid limits for weak solutions are in general hard to obtain, and in this case we only obtain a weak $L^{2}$ result without a rate. We believe that explicit rates can be given in certain negative Sobolev norms.

The second part is devoted to regularity estimates for the dissipative QGS equations. Although we use the ideas of Foias-Temam [8] and Doering-Titi [7] developed for the Navier-Stokes equations, our methods of estimating the non-linear terms are significantly different from theirs because of the special structure of the QGS equations. We treat the norm $\left\|e^{\gamma t \Lambda^{\alpha}} \Lambda^{\beta} \vartheta\right\|_{L^{2}}$ (where $\Lambda=$ $(-\Delta)^{1 / 2}$ - see notations in the following sections), and a special consequence of our estimates for $\left\|e^{\gamma t \Lambda^{\alpha}} \Lambda^{\beta} \vartheta\right\|_{L^{2}}$ is that, as long as $\left\|\Lambda^{\beta} \vartheta\right\|_{L^{2}}^{2}$ remains bounded, the Fourier spectrum decays exponentially at high wave numbers. We note here that $\kappa\left\|\Lambda^{\beta} \vartheta\right\|_{L^{2}}^{2}$ for $\beta=1$ is the analogue of the energy dissipation rate of the 3 -D Navier-Stokes equations. In a similar fashion as Doering and Titi [7] argue for the Navier-Stokes equations, these exponential decay estimates can be used to obtain bounds on small length scale defined through the exponential decay rate. 
Here I would like to thank Professor Peter Constantin for his suggestions and help.

2. Inviscid limits. We consider the $2-\mathrm{D}$ dissipative $(\kappa>0)$ and the nondissipative $(\kappa=0)$ quasi-geostrophic equations on $\mathbb{R}^{2}$ or $\mathbb{T}^{2}$ :

$$
\frac{\partial \vartheta}{\partial t}+u \cdot \nabla \vartheta+\kappa \Lambda^{2 \alpha} \vartheta=0, \quad 0 \leq \alpha \leq 1
$$

where $\Lambda=(-\Delta)^{1 / 2}$ is the Riesz potential operator and the velocity $u$ is defined from the stream function $\psi=-\Lambda^{-1} \vartheta$ by

$$
u=\left(u_{1}, u_{2}\right)=\left(-\frac{\partial \psi}{\partial x_{2}}, \frac{\partial \psi}{\partial x_{1}}\right)
$$

We use $\mathbb{D}^{2}$ to denote either $\mathbb{R}^{2}$ or $\mathbb{T}^{2}$. We first consider the smooth initial data case: $\vartheta_{0} \in H^{k}\left(\mathbb{D}^{2}\right)(k \geq 3)$. As shown by Constantin, Majda and Tabak [3], the QGS equations with smooth initial data have local (in time) smooth solutions and the Beale-Kato-Majda type blowup conditions have been obtained. More precisely, we state the following result:

Proposition 2.1 If the initial data $\left.\vartheta\right|_{t=0}=\vartheta_{0} \in H^{k}\left(\mathbb{D}^{2}\right)$ for some $k \geq 3$, then both the QGS and the dissipative QGS equations have a unique smooth solution for a small time interval, respectively. Furthermore, the solution $\vartheta^{Q G}$ of the QGS equations satisfies

$$
\begin{gathered}
\int_{0}^{t}\left\|\nabla \vartheta^{Q G}(\cdot, s)\right\|_{L^{\infty}} d s<\infty \\
\int_{0}^{t}\left\|\vartheta^{Q G}(\cdot, s)\right\|_{k}^{2} d s<\infty
\end{gathered}
$$

for any $t$ belonging to the existence interval $\left[0, T^{*}\right)$.

This proposition admits the possibility of finite-time singularity formation and consequently, the inviscid limit results for the smooth solutions are valid only for the time period before the possible breakdown. We need further estimates on the solutions.

Proposition 2.2 Let $\vartheta^{Q G}$ and $\vartheta^{D Q G}$ be the smooth solutions of the $Q G S$ and the dissipative $Q G S$ equations with the same initial data $\vartheta_{0} \in H^{k}\left(\mathbb{D}^{2}\right)(k \geq$ 3). $u^{Q G}$ and $u^{D Q G}$ are the corresponding velocities and $\psi^{Q G}$ and $\psi^{D Q G}$ are the stream functions, respectively. Then for any $t$ in the maximal time interval $\left[0, T^{*}\right)$ (when the smooth solutions exist), 
(1) The solution $\vartheta^{Q G}$ of the QGS equation satisfies

$$
\int_{\mathbb{D}^{2}} G\left(\vartheta^{Q G}(x, t)\right) d x=\int_{\mathbb{D}^{2}} G\left(\vartheta_{0}\right) d x,
$$

where $G$ is a continuous function with $G(0)=0$. Especially,

$$
\left\|\vartheta^{Q G}(\cdot, t)\right\|_{L^{p}}=\left\|\vartheta_{0}\right\|_{L^{p}}, \quad 1 \leq p \leq \infty .
$$

Furthermore,

$$
\begin{aligned}
& \left\|u^{Q G}(\cdot, t)\right\|_{L^{2}}=\left\|\vartheta^{Q G}(\cdot, t)\right\|_{L^{2}}=\left\|\vartheta_{0}\right\|_{L^{2}}, \\
& \left\|u^{Q G}(\cdot, t)\right\|_{L^{q}} \leq C_{q}\left\|\vartheta^{Q G}(\cdot, t)\right\|_{L^{q}}, \quad 1<q<\infty,
\end{aligned}
$$

where $C_{q}$ is a constant depending on $q$.

(2) The solution $\vartheta^{D Q G}$ of the dissipative QGS equation obeys

$$
\begin{aligned}
& \left\|\vartheta^{D Q G}(\cdot, t)\right\|_{L^{p}} \leq\left\|\vartheta_{0}\right\|_{L^{p}}, \quad 1 \leq p \leq \infty, \\
& \left\|u^{D Q G}(\cdot, t)\right\|_{L^{q}} \leq C_{q}\left\|\vartheta^{D Q G}(\cdot, t)\right\|_{L^{q}}, \quad 1<q<\infty .
\end{aligned}
$$

Proof. The proof of this proposition is classical. The $L^{q}$ estimates are the classical inequality for the Calderon-Zygmund singular integrals, while the $L^{p}$ bounds come from energy estimates. We omit the details.

We now state the inviscid limit theorem for smooth solutions.

Theorem 2.3 Let $\vartheta^{Q G}$ and $\vartheta^{D Q G}$ be the smooth solutions of the $Q G S$ equations and the dissipative QGS equations with the same initial data $\vartheta_{0} \in$ $H^{k}\left(\mathbb{D}^{2}\right)(k \geq 3)$. If $\left[0, T^{*}\right)$ is the maximal time interval of smooth existence, then for any $t<T^{*}$,

$$
\left\|\vartheta^{Q G}(\cdot, t)-\vartheta^{D Q G}(\cdot, t)\right\|_{L^{2}\left(\mathbb{D}^{2}\right)} \leq C \kappa,
$$

where $C$ is a constant depending on $\vartheta_{0}$ and $T^{*}$ only.

The convergence rate $O(\kappa)$ is optimal. In the proof we only treat the case $\mathbb{D}^{2}=\mathbb{R}^{2}$; the case $\mathbb{D}^{2}=\mathbb{T}^{2}$ is easier.

Proof of Theorem 2.3. Consider the difference

$$
\vartheta(x, t)=\vartheta^{D Q G}(x, t)-\vartheta^{Q G}(x, t)
$$

between the solutions of the QGS and the dissipative QGS equations, and let $u(x, t)$ be the corresponding velocity difference. This difference $\vartheta$ satisfies

$$
\frac{\partial \vartheta}{\partial t}+u^{D Q G} \cdot \nabla \vartheta+u \cdot \nabla \vartheta^{Q G}+\kappa \Lambda^{2 \alpha}\left(\vartheta+\vartheta^{Q G}\right)=0,
$$


where $\Lambda^{2 \alpha}=(-\Delta)^{\alpha}$. Multiplying by $\vartheta$ and integrating in space, we obtain

$$
\frac{1}{2} \frac{d}{d t} \int \vartheta^{2} d x+\kappa \int\left(\Lambda^{2 \alpha} \vartheta\right) \vartheta d x=\mathrm{I}+\mathrm{II}+\mathrm{III}
$$

where

$$
\begin{aligned}
\mathrm{I} & =\int\left(u^{D Q G} \cdot \nabla \vartheta\right) \vartheta d x, \\
\mathrm{II} & =\int\left(u \cdot \nabla \vartheta^{Q G}\right) \vartheta d x, \\
\mathrm{III} & =\kappa \int\left(\Lambda^{2 \alpha} \vartheta^{Q G}\right) \vartheta d x .
\end{aligned}
$$

We estimate these three terms and start with the first one. Clearly the estimates in Proposition 2.2 guarantee that $\mathrm{I}$ is integrable. We show that it is actually zero. Let $\chi$ be a smooth cut-off function: $\chi(x)=1$ if $|x|<1$ and $\chi(x)=0$ if $|x|>2$ and $\chi_{r}=\chi(x / r)$ for $r>0$. Using the Dominated Convergence Theorem and the Divergence Theorem,

$$
\begin{aligned}
\mathrm{I} & =\lim _{r \rightarrow \infty} \int\left(u^{D Q G} \cdot \nabla \vartheta\right) \vartheta \chi_{r}(x) d x \\
& =-\lim _{r \rightarrow \infty} \frac{1}{2 r} \int \chi^{\prime} \cdot u^{D Q G} \vartheta^{2} d x
\end{aligned}
$$

since the last integral is bounded,

$$
\begin{aligned}
\left|\int \chi^{\prime} \cdot u^{D Q G} \vartheta^{2} d x\right| \leq \int\left|u^{D Q G}\right| \vartheta^{2} d x & \leq\left\|\vartheta^{D Q G}\right\|_{L^{2}}\|\vartheta\|_{L^{4}}^{2} \\
& \leq 4\left\|\vartheta_{0}\right\|_{L^{2}}\left\|\vartheta_{0}\right\|_{L^{4}}^{2}
\end{aligned}
$$

we obtain $\mathrm{I}=0$. II and III can be estimated by using Proposition 2.2,

$$
\begin{aligned}
& |\mathrm{II}| \leq\left\|\nabla \vartheta^{Q G}\right\|_{L^{\infty}}\|u\|_{L^{2}}\|\vartheta\|_{L^{2}}=\left\|\nabla \vartheta^{Q G}\right\|_{L^{\infty}}\|\vartheta\|_{L^{2}}^{2}, \\
& |\mathrm{III}| \leq \frac{\kappa^{2}}{2} \int\left(\Lambda^{2 \alpha} \vartheta^{Q G}\right)^{2} d x+\frac{1}{2} \int \vartheta^{2} d x .
\end{aligned}
$$

Collecting these estimates, we obtain

$$
\frac{d}{d t} \int \vartheta^{2} d x+\kappa\left\|\Lambda^{\alpha} \vartheta\right\|_{L^{2}}^{2} d x \leq \mathcal{P}(t)\|\vartheta\|_{L^{2}}^{2}+\kappa^{2}\left\|\vartheta^{Q G}\right\|_{2 \alpha}^{2}
$$

where

$$
\mathcal{P}(t)=2\left\|\nabla \vartheta^{Q G}(\cdot, t)\right\|_{L^{\infty}}+1 .
$$

By Gronwall's inequality,

$$
\|\vartheta\|_{L^{2}}^{2} \leq e^{\int_{0}^{t} \mathcal{P}(s) d s}\left\|\vartheta_{0}\right\|_{L^{2}}^{2}+\kappa^{2} \int_{0}^{t} e^{\int_{\tau}^{t} \mathcal{P}(s) d s}\left\|\vartheta^{Q G}\right\|_{2 \alpha}^{2} d \tau .
$$


Noting that $\vartheta_{0}=0$ and using the result of Proposition 2.1, especially,

$$
\begin{array}{r}
\int_{0}^{t}\left\|\nabla \vartheta^{Q G}(\cdot, s)\right\|_{L^{\infty}} d s<\infty \\
\int_{0}^{t}\left\|\vartheta^{Q G}(\cdot, s)\right\|_{k}^{2} d s<\infty
\end{array}
$$

we obtain

$$
\|\vartheta\|_{L^{2}} \leq C \kappa
$$

which completes the proof of Theorem 2.3.

We now turn to weak solutions of these equations corresponding to $L^{2}$ initial data. We restrict ourselves to the periodic domain $\mathbb{T}^{2}=[0, L] \times[0, L]$. We quote the result of Resnick [11] on the existence of weak solutions.

Proposition 2.4 Let $\vartheta_{0} \in L^{2}\left(\mathbb{T}^{2}\right)$ and $T>0$ be arbitrarily fixed. Then there exist weak solutions

$$
\begin{aligned}
\vartheta^{Q G} & \in L^{\infty}\left([0, T] ; L^{2}\left(\mathbb{T}^{2}\right)\right), \\
\vartheta^{D Q G} & \in L^{\infty}\left([0, T] ; L^{2}\left(\mathbb{T}^{2}\right)\right) \cap L^{2}\left([0, T] ; H^{\alpha}\left(\mathbb{T}^{2}\right)\right)
\end{aligned}
$$

of the QGS and the dissipative QGS equations, respectively. That is, for each test function $\varphi \in C^{\infty}\left(\mathbb{T}^{2}\right)$,

$$
\begin{array}{r}
\int \vartheta^{Q G} \varphi d x-\int \vartheta_{0} \varphi d x-\int_{0}^{T} \int_{\mathbb{T}^{2}} \vartheta^{Q G}\left(u^{Q G} \cdot \nabla \varphi\right) d x d t=0, \\
\int \vartheta^{D Q G} \varphi d x-\int \vartheta_{0} \varphi d x-\int_{0}^{T} \int_{\mathbb{T}^{2}} \vartheta^{D Q G}\left(u^{D Q G} \cdot \nabla \varphi\right) d x d t=0,
\end{array}
$$

where $u^{Q G}$ and $u^{D Q G}$ are the velocities corresponding to $\vartheta^{Q G}$ and $\vartheta^{D Q G}$.

These weak solutions are constructed by using classical Galerkin approximations. The weak $L^{2}$ inviscid limit result is an easy consequence of this construction method.

Theorem 2.5 Let $\vartheta_{0} \in L^{2}\left(\mathbb{T}^{2}\right)$ and $\vartheta^{Q G}$ and $\vartheta^{D Q G}$ be the weak solutions of the QGS and the dissipative QGS equations with the same initial data $\vartheta_{0}$. Then for any arbitrarily fixed $T>0$ and any $\varphi \in L^{2}\left(\mathbb{T}^{2}\right)$,

$$
\limsup _{\kappa \rightarrow 0}\left(\vartheta^{D Q G}(\cdot, t)-\vartheta^{Q G}(\cdot, t), \varphi\right)=0, \quad \text { for any } t \leq T .
$$

Proof. Consider the $n^{\text {th }}$ Galerkin approximations $\left\{\vartheta_{n}^{Q G}\right\}$ and $\left\{\vartheta_{n}^{D Q G}\right\}$, which are in the space $S_{n}$ spanned by the Fourier modes $e^{i m x}$ with $0<|m| \leq n$ and satisfy

$$
\frac{\partial \vartheta_{n}}{\partial t}+P_{n}\left(u_{n} \cdot \nabla \vartheta_{n}\right)+\kappa \Lambda^{2 \alpha} \vartheta_{n}=0
$$




$$
\left.\vartheta_{n}\right|_{t=0}=P_{n} \vartheta_{0}
$$

where $P_{n}$ is the orthogonal projection from $L^{2}$ onto $S_{n}$ and $\kappa=0$ in the case of $\vartheta^{Q G}$. As we know from [11], for some subsequences $\vartheta_{n}^{D Q G} \rightarrow \vartheta^{D Q G}$,

$$
\vartheta_{n}^{Q G} \rightarrow \vartheta^{Q G} \quad \text { weakly in } L^{2}\left(\mathbb{T}^{2}\right)
$$

So, taking large $n$, we have for any $\varepsilon>0$

$$
\begin{aligned}
\left|\left(\vartheta^{D Q G}(\cdot, t)-\vartheta^{Q G}(\cdot, t), \varphi\right)\right| & \leq \varepsilon+\left|\left(\vartheta_{n}^{D Q G}-\vartheta_{n}^{Q G}, \varphi\right)\right| \\
& \leq \varepsilon+\|\varphi\|_{L^{2}}\left\|\vartheta_{n}^{D Q G}-\vartheta_{n}^{Q G}\right\|_{L^{2}} \\
& \leq \varepsilon+C_{n} \kappa,
\end{aligned}
$$

which implies (2). Here we've applied the inviscid limit result for smooth solutions to $\vartheta_{n}^{D Q G}-\vartheta_{n}^{Q G}$.

Remark 2.6 Since the constants $C_{n}$ in the inequality (3) depends on $n$, we obtain no convergence rate. An explicit rate may exist in negative Sobolev norms.

3. Regularity estimates. We consider the 2-D dissipative QGS equations with smooth initial data on the torus $\mathbb{T}^{2}=[0, L] \times[0, L]$, which admits a unique classical local solution. Let $\vartheta$ be this solution. We will estimate the quantity

$$
\left\|e^{\gamma t \Lambda^{\alpha}} \Lambda^{\beta} \vartheta\right\|_{L^{2}}^{2}
$$

where the operators $\Lambda^{\beta}$ and $e^{\lambda \Lambda^{\alpha}}$ are defined through the Fourier transform

$$
\begin{gathered}
\Lambda^{\beta} f=L^{-2} \sum_{k} e^{i k \cdot x}|k|^{\beta} \widehat{f}(k), \\
e^{\lambda \Lambda^{\alpha}} f=L^{-2} \sum_{k} e^{i k \cdot x+\lambda|k|^{\alpha}} \widehat{f}(k),
\end{gathered}
$$

with $\widehat{f}(k)$ being the $k^{\text {th }}$ Fourier mode of $f$,

$$
\widehat{f}(k)=\int_{\mathbb{T}^{2}} e^{-i k \cdot x} f(x) d x .
$$

It is easy to see from these notations that $e^{\lambda \Lambda^{\alpha}}$ commutes with $\Lambda^{\beta}$ and the partial derivatives for periodic boundary conditions considered here.

We obtain bounds for the quantity $\left\|e^{\gamma t \Lambda^{\alpha}} \Lambda^{\beta} \vartheta\right\|_{L^{2}}^{2}$, which lead to the exponential decay of the Fourier spectrum of $\vartheta$. The precise estimates are as follows.

Theorem 3.7 Consider the 2-D dissipative QGS equations

$$
\frac{\partial \vartheta}{\partial t}+u \cdot \nabla \vartheta+\kappa \Lambda^{2 \alpha} \vartheta=0, \quad \kappa>0, \frac{1}{2}<\alpha \leq 1,
$$


on the 2-D torus $\mathbb{T}^{2}=[0, L] \times[0, L]$. Let the initial data $\vartheta_{0} \in H^{k}\left(\mathbb{T}^{2}\right)$ with mean zero and $\vartheta$ be the unique smooth solution. We take $\beta$ such that

$$
\beta>0, \quad \beta+2 \alpha>2 .
$$

Then for any $\gamma>0, \vartheta$ satisfies

(5) $\left\|e^{\gamma t \Lambda^{\alpha}} \Lambda^{\beta} \vartheta\right\|_{L^{2}}^{2}$

$$
\leq \frac{e^{2 \gamma^{2} t / \kappa}\left\|\Lambda^{\beta} \vartheta_{0}\right\|_{L^{2}}^{2}}{\left(1-C\left(\left\|\Lambda^{\beta} \vartheta_{0}\right\|_{L^{2}}^{2}\right)^{N-1} \kappa^{-(M-1)} \gamma^{-2}\left(e^{2\left(N_{1}\right) \gamma^{2} t / \kappa}-1\right)\right)^{1 /(N-1)}},
$$

which is finite for $t \in\left[0, t^{*}\right)$,

$$
t^{*}=\frac{\kappa}{2(N-1) \gamma^{2}} \log \left(1+\frac{\kappa^{M-1} \gamma^{2}}{C\left\|\Lambda^{\beta} \vartheta_{0}\right\|_{L^{2}}^{2(N-1)}}\right) .
$$

Here $C$ is a constant and $M, N$ are given by

$$
\begin{aligned}
M & =\frac{\alpha+\sigma}{\alpha-\sigma}, \\
N & =1+\frac{\alpha}{\alpha-\sigma}, \\
\sigma & = \begin{cases}1-\alpha, & \text { if } \beta \geq 1 \\
2-\beta-\alpha, & \text { if } \beta \leq 1\end{cases}
\end{aligned}
$$

A special consequence of this theorem is that each Fourier mode amplitude can be individually controlled. In fact, a rough estimate gives

$$
e^{2 \gamma t|k|^{\alpha}}|k|^{2 \beta}|\vartheta(k, t)|^{2} \leq \sum_{k} e^{2 \gamma t|k|^{\alpha}}|k|^{2 \beta}|\vartheta(k, t)|^{2}=L^{2}\left\|e^{\gamma t \Lambda^{\alpha}} \Lambda^{\beta} \vartheta\right\|_{L^{2}}^{2} .
$$

Thus the $k^{\text {th }}$ mode is bounded by

$|\vartheta(k, t)|^{2} \leq \frac{L^{2}}{|k|^{2 \beta}} \frac{e^{\left(\gamma^{2} t / \kappa\right)-2 \gamma t|k|^{\alpha}}\left\|\Lambda^{\beta} \vartheta_{0}\right\|_{L^{2}}^{2}}{\left(1-C\left(\left\|\Lambda^{\beta} \vartheta_{0}\right\|_{L^{2}}^{2}\right)^{N-1} \kappa^{-(M-1)} \gamma^{-2}\left(e^{2(N-1) \gamma^{2} t / \kappa}-1\right)\right)^{1 /(N-1)}}$

for $t \in\left[0, t^{*}\right)$.

Doering and Titi [7] establish the exponential decay of the power spectrum for the flow field of the 3-D Navier-Stokes equations. A similar analysis based on these bounds can be made to conclude that if $\left\|\Lambda^{\beta} \vartheta\right\|_{L^{2}}^{2}$ is bounded uniformly in time, then after a transient time of length $t^{*} / 2$ the Fourier spectrum of $\vartheta$ decays exponentially at high wave numbers. Furthermore, the associated decay length can be defined and estimated in terms of the dissipation rates.

The main difficulty in proving the estimate (5) is how to bound the non-linear term properly. We need the inequalities for the Calderon-Zygmund type singular integrals. We'll also use the following lemma concerning the operator $\Lambda^{s}$, which is proved in [11], [13]. 
Lemma 3.8 For $s>0$ and $1<r<p \leq \infty$,

$$
\left\|\Lambda^{s}(u v)\right\|_{L^{r}} \leq C\left(\|u\|_{L^{p}}\left\|\Lambda^{s} v\right\|_{L^{q}}+\|v\|_{L^{p}}\left\|\Lambda^{s} u\right\|_{L^{q}}\right)
$$

where $1 / r=1 / p+1 / q$ and $C$ is a constant.

Proof of Theorem 3.1Using Equation (4), we obtain

$$
\begin{aligned}
\frac{1}{2} \frac{d}{d t} \int\left|e^{\gamma t \Lambda^{\alpha}} \Lambda^{\beta} \vartheta\right|^{2} d x & =\int\left(e^{\gamma t \Lambda^{\alpha}} \Lambda^{\beta} \vartheta\right)\left(\gamma e^{\gamma t \Lambda^{\alpha}} \Lambda^{\alpha} \Lambda^{\beta} \vartheta+e^{\gamma t \Lambda^{\alpha}} \Lambda^{\beta} \frac{\partial \vartheta}{\partial t}\right) d x \\
& =\mathrm{I}+\mathrm{II}+\mathrm{III},
\end{aligned}
$$

where

$$
\begin{aligned}
\mathrm{I} & =\gamma \int\left(e^{\gamma t \Lambda^{\alpha}} \Lambda^{\beta} \vartheta\right)\left(e^{\gamma t \Lambda^{\alpha}} \Lambda^{\alpha} \Lambda^{\beta} \vartheta\right) d x \\
& \leq \frac{\gamma^{2}}{\kappa}\left\|e^{\gamma t \Lambda^{\alpha}} \Lambda^{\beta} \vartheta\right\|_{L^{2}}^{2}+\frac{\kappa}{4}\left\|e^{\gamma t \Lambda^{\alpha}} \Lambda^{\alpha+\beta} \vartheta\right\|_{L^{2}}^{2} \\
\mathrm{II} & =-\int\left(e^{\gamma t \Lambda^{\alpha}} \Lambda^{\beta} \vartheta\right)\left(e^{\gamma t \Lambda^{\alpha}} \Lambda^{\beta}(u \cdot \nabla \vartheta)\right) d x \\
\mathrm{III} & =-\kappa \int\left(e^{\gamma t \Lambda^{\alpha}} \Lambda^{\beta} \vartheta\right)\left(e^{\gamma t \Lambda^{\alpha}} \Lambda^{2 \alpha+\beta} \vartheta\right) d x \\
& =-\kappa\left\|e^{\gamma t \Lambda^{\alpha}} \Lambda^{\alpha+\beta} \vartheta\right\|_{L^{2}}^{2} .
\end{aligned}
$$

Now we deal with the second term II. Since the operators commute,

$$
\mathrm{II}=-\int\left(\Lambda^{(\alpha+\beta)} e^{\gamma t \Lambda^{\alpha}} \vartheta\right)\left(R \Lambda^{\beta-\alpha+1} e^{\gamma t \Lambda^{\alpha}}(u \cdot \vartheta)\right) d x
$$

where $R=\left(\partial_{x_{1}} \Lambda^{-1}, \partial_{x_{2}} \Lambda^{-1}\right)$ are Riesz transforms. For brevity, we'll use the notations

$$
\tilde{u}=e^{\gamma t \Lambda^{\alpha}} u, \quad \tilde{\vartheta}=e^{\gamma t \Lambda^{\alpha}} \vartheta .
$$

To obtain further estimates, we break up the term $e^{\gamma t \Lambda^{\alpha}}(u \cdot \vartheta)$. Its structure can be better seen from the Fourier transform:

$$
e^{\gamma t \Lambda^{\alpha}}(u \cdot \vartheta)=L^{-2} \sum_{k^{\prime}+k^{\prime \prime}=k} e^{\gamma t|k|^{\alpha}} \widehat{u}\left(k^{\prime}\right) \widehat{\vartheta}\left(k^{\prime \prime}\right)
$$

For $0 \leq \alpha \leq 1$,

$$
|k|^{\alpha}=\left|k^{\prime}+k^{\prime \prime}\right|^{\alpha} \leq\left|k^{\prime}\right|^{\alpha}+\left|k^{\prime \prime}\right|^{\alpha},
$$

so this term is bounded by

$$
L^{-2} \sum_{k^{\prime}} e^{\gamma t\left|k^{\prime}\right|^{\alpha}}\left|u\left(k^{\prime}\right)\right| \cdot \sum_{k^{\prime \prime}} e^{\gamma t\left|k^{\prime \prime}\right|^{\alpha}}\left|\vartheta\left(k^{\prime \prime}\right)\right|
$$


Now, using Hölder's inequality and the boundedness of the Riesz transform, we have

$$
|\mathrm{II}| \leq C\|\tilde{\vartheta}\|_{H^{\alpha+\beta}}\left\|\Lambda^{\beta+1-\alpha}(\tilde{u} \cdot \tilde{\vartheta})\right\|_{L^{2}},
$$

where $C$ is a constant.

Now we take $q=2$ if $\beta>1$ and $q=2 / \beta$ if $\beta \leq 1$. Choose $p$ such that

$$
\frac{2}{p}+\frac{2}{q}=1
$$

and $\sigma=2-2 / q-\alpha$. The condition that $\beta+2 \alpha>2$ implies that $0<\sigma<\alpha$. We apply Lemma 2.2 to obtain

$$
|\mathrm{II}| \leq C\|\tilde{\vartheta}\|_{H^{\alpha+\beta}}\left(\|\tilde{u}\|_{L^{p}}\left\|\Lambda^{\beta+1-\alpha} \tilde{\vartheta}\right\|_{L^{q}}+\|\tilde{\vartheta}\|_{L^{p}}\left\|\Lambda^{\beta+1-\alpha} \tilde{u}\right\|_{L^{q}}\right)=\mathrm{II}_{1}+\mathrm{II}_{2} .
$$

Using Sobolev imbeddings, we have

$$
H^{\beta} \hookrightarrow H^{\frac{2}{q}} \hookrightarrow L^{p}, \quad H^{\beta+\sigma} \hookrightarrow L_{\beta+1-\alpha}^{p},
$$

and from the Gagliado-Nirenberg interpolation (since $\sigma<\alpha$ )

$$
\beta+\sigma=\frac{\sigma}{\alpha}(\beta+\alpha)+\left(1-\frac{\sigma}{\alpha}\right) \beta,
$$

we have

$$
\begin{aligned}
\mathrm{II}_{1} & \leq C\|\tilde{\vartheta}\|_{H^{\beta+\alpha}}\|\tilde{u}\|_{H^{\beta}}\|\tilde{\vartheta}\|_{H^{\beta+\sigma}} \\
& \leq C\|\tilde{\vartheta}\|_{H^{\beta}}\|\tilde{\vartheta}\|_{H^{\beta+\alpha}}\|\tilde{\vartheta}\|_{H^{\beta+\sigma}} \\
& \leq C\|\tilde{\vartheta}\|_{H^{\beta+\alpha}}^{1+\sigma / \alpha}\|\tilde{\vartheta}\|_{H^{\beta}}^{2-\sigma / \alpha}
\end{aligned}
$$

where $C$ is constant depending on $\alpha$ and $\beta$. By Young's inequality

$$
\mathrm{II}_{1} \leq \frac{\kappa}{8}\|\tilde{\vartheta}\|_{H^{\beta+\alpha}}^{2}+\frac{C}{\kappa^{M}}\left(\|\tilde{\vartheta}\|_{\beta}^{2}\right)^{N}
$$

where $N=(2 \alpha-\sigma) /(\alpha-\sigma)$ and $M=(\alpha+\sigma) /(\alpha-\sigma)$.

A similar estimate results in the same bound for $\mathrm{II}_{2}$.

Collecting the estimates for I, II, and III and reintroducing $\tilde{\vartheta}=e^{\gamma t \Lambda^{\alpha}} \vartheta$, we have

$$
\begin{aligned}
\frac{d}{d t}\left\|e^{\gamma t \Lambda^{\alpha}} \Lambda^{\beta} \vartheta\right\|_{L^{2}}^{2} \leq-\kappa\left\|e^{\gamma t \Lambda^{\alpha}} \Lambda^{\beta+\alpha} \vartheta\right\|_{L^{2}}^{2} & \\
& +\frac{2 \gamma^{2}}{\kappa}\left\|e^{\gamma t \Lambda^{\alpha}} \Lambda^{\beta} \vartheta\right\|_{L^{2}}^{2}+\frac{C}{\kappa^{M}}\left\|e^{\gamma t \Lambda^{\alpha}} \Lambda^{\beta} \vartheta\right\|_{L^{2}}^{2 N} .
\end{aligned}
$$

As we let

$$
Z(t)=\left\|e^{\gamma t \Lambda^{\alpha}} \Lambda^{\beta} \vartheta\right\|_{L^{2}}^{2},
$$


the differential inequality becomes

$$
\frac{d Z}{d t} \leq \frac{2 \gamma^{2}}{\kappa} Z+\frac{C}{\kappa^{M}} Z^{N}
$$

Elementary algebraic calculations yield

$$
\frac{d Y}{d t} \leq \frac{C e^{2(N-1)\left(\gamma^{2} t / \kappa\right)}}{\kappa^{M}} Y^{N}
$$

where $Y=e^{-2 \gamma^{2} t / \kappa} Z$. After a simple calculation,

$$
Y \leq \frac{Y_{0}}{\left(1-C Y_{0}^{N-1} \kappa^{-(M-1)} \gamma^{-2}\left(e^{2(N-1) \gamma^{2} t / \kappa}-1\right)\right)^{1 /(N-1)}}
$$

Reintroducing $Z(t)=\left\|e^{\gamma t \Lambda^{\alpha}} \Lambda^{\beta} \vartheta\right\|_{2}^{2}$ and noting that $Z(0)=\left\|\Lambda^{\beta} \vartheta_{0}\right\|_{2}^{2}$, we have that

$$
\left\|e^{\gamma t \Lambda^{\alpha}} \Lambda^{\beta} \vartheta\right\|_{2}^{2} \leq \frac{e^{2 \gamma^{2} t / \kappa}\left\|\Lambda^{\beta} \vartheta_{0}\right\|_{2}^{2}}{\left(1-C\left(\left\|\Lambda^{\beta} \vartheta_{0}\right\|_{2}^{2}\right)^{N-1} \kappa^{-(M-1)} \gamma^{-2}\left(e^{2(N-1) \gamma^{2} t / \kappa}-1\right)\right)^{1 /(N-1)}} .
$$

This means that $\left\|e^{\gamma t \Lambda^{\alpha}} \Lambda^{\beta} \vartheta\right\|_{2}^{2}$ is finite on the interval $\left[0, t^{*}\right)$, where

$$
t^{*}=\frac{\kappa}{2(N-1) \gamma^{2}} \log \left(1+\frac{\kappa^{M-1} \gamma^{2}}{C\left\|\Lambda^{\beta} \vartheta_{0}\right\|_{2}^{2(N-1)}}\right)
$$

The smaller the initial decay rate $\left\|\Lambda^{\beta} \vartheta_{0}\right\|_{2}$ is, the larger $t^{*}$. Similarly, the larger the parameter $\gamma$, the shorter $t^{*}$.

\section{REFERENCES}

[1] J. T. BeAle, A. MAJDA, Rates of convergence for viscous splitting of the NavierStokes equations, Math. Comp. 37 (1981), 243-259.

[2] P. Constantin, Note on loss of regularity for solutions of the $3 D$ incompressible Euler and related equations, Commun. Math. Phys., 104 (1986), 311-326.

[3] P. Constantin, A. Majda, E. Tabak, Formation of strong fronts in the 2-D quasi-geostrophic thermal active scalar, Nonlinearity, 7(1994), 1495-1533

[4] P. Constantin, J. Wu, Inviscid limit for vortex patches, Nonlinearity, 8(1995), 735-742

[5] P. Constantin, J. Wu, Vanishing viscosity limit for vortex patches, Institut Mittag-Leffler, No. 26(1995)

[6] P. Constantin, J. Wu, The inviscid limit for non-smooth vorticity, Indiana Univ. Math. J., 45(1996), 67-81

[7] Ch. Doering, E. Titi, Exponential decay rate of the power spectrum for solutions of the Navier-Stokes equations, Phys. of Fluids, 7 (1995), 1384-1390.

[8] C. Foias, R. Temam, Gevrey class regularity for the solutions of the NavierStokes equations, J. Funct. Anal., 87(1989), 359-369. 
[9] I. Held, R. Pierrehumbert, S. Garner, K. Swanson, Surface quasigeostrophic dynamics, J. Fluid Mech., 282(1995), 1-20

[10] J. Pedlosky, Geophysical Fluid Dynamics, Springer, New York, 1987

[11] S. Resnick, Dynamical Problems in Non-linear Advective Partial Differential Equations, Ph.D. thesis, University of Chicago, 1995

[12] E. TABAK, Quasi-geostrophic Equations, Proceedings of the Ohio State University Conference on Navier-Stokes, Euler equations and Singularities, Ohio, 1997.

[13] M. TAYLOR, Pseudodifferential Operators and Nonlinear PDE, Birkhäuser, 1993

This work was supported by grant DMS 9304580 from the National Science Foundation.

Department of Mathematics

The University of Chicago

Chicago, Illinois 60637

Current Address:

Department of Mathematics

The University of Texas at Austin

RLM 8.100

Austin, Texas 78712

Received: May 9th, 1996; revised: September 3rd, 1997; 